\title{
Design, construction and characterization of a portable irradiator to calibrate installed ambient dose equivalent monitors
}

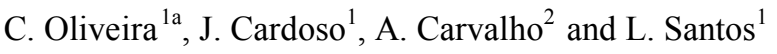 \\ ${ }^{1}$ Laboratório de Metrologia das Radiações Ionizantes, Instituto Superior Técnico, Pólo de Loures. Est. Nac. No ${ }^{\circ} 10$, \\ 2686-953 Sacavém, Portugal. \\ ${ }^{2}$ Faculdade de Ciências e Tecnologia da Universidade Nova de Lisboa, Monte da Caparica, Portugal
}

\begin{abstract}
Calibration of installed dose monitors measuring the ambient dose equivalent, $H^{*}(10)$, should be done periodically according to portuguese national law. These monitors are fixed on the wall of the facility, hospitals or any other installation and usually they are checked in situ. To be able to realize this calibration LMRI (Metrology Laboratory for Ionizing Radiation) constructed a portable irradiator made of lead with a ${ }^{137} \mathrm{Cs}$ source. The dosimetric characterization of the irradiator was realized and the $H^{*}(10)$ was determined at several distances from the irradiator. The profiles at different distances from the irradiator have been performed either experimentally or using the MCNPX code and was observed that the full width at half maximum of the radiation field is a linear function of the distance to the irradiator. The influence of the wall where the monitors are installed was studied by Monte Carlo simulation and correction factors applied to the $H^{*}(10)$ are determined.
\end{abstract}

\section{Introduction}

According to the European Council Directive 986/29/Euratom [1] and the new version already approved by European Parliament, the European metrology laboratories must be able to calibrate and control dosemeters in terms of operational radiation protection quantities. According to Portuguese law, of the radiation protection dose monitors measuring the quantity ambient dose equivalent $H^{*}(10)$, should be checked periodically, with a periodicity of two years. These monitors can be portable or fixed (installed) on the wall of the facility, hospitals or any other facilities, and for these situations they are usually checked in situ. So, for this purpose, the LMRI (Metrology Laboratory for Ionizing Radiation) constructed a portable irradiator made of lead and equipped with a ${ }^{137} \mathrm{Cs}$ source, which is the reference radiation for calibration purposes of these radiation protection devices according to IEC 60532 [2]. The design of the irradiator considered two parameters of contrary sense, its weight and the dose at its exterior surface. The weight is an important parameter, because its use requires that it is hand-held. After its construction the radiation field was characterized and the dosimetry was realized. Finally, the influence of the wall where the monitors are usually fixed has been studied by Monte Carlo and correction factors determined.

\section{Design of the irradiator}

The ${ }^{137} \mathrm{Cs}$ source used [3], has an air kerma rate at $1 \mathrm{~m}$ (10 $\mathrm{cm})$ distance of $14 \mu \mathrm{Gy} / \mathrm{h}(1.4 \mathrm{mGy} / \mathrm{h})$ and an estimated activity of $0.18 \mathrm{GBq}$ at the reference date. The source has the spherical shape and it is involved in an acrylic material as it is shown in figure 1 .

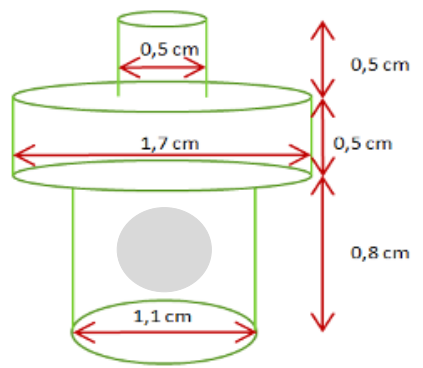

Figure 1.The ${ }^{137} \mathrm{Cs}$ source with the spherical shape (in grey) inside the acrylic covering.

To calculate the ambient dose equivalent the conversion coefficient $1.20 \mathrm{~Sv} / \mathrm{Gy}$ was used [4].

Taking to account the dose limit $20 \mathrm{mSv} /$ year, a decision was taken to establish the dose when the source will be inside the irradiator. This dose should take into account the number of working days with the irradiator.

\footnotetext{
$\overline{{ }^{\mathrm{a}} \text { Corresponding author: coli@ctn.ist.utl.pt }}$
} 
Taking to account that (i) during the control of the radiation protection monitors, the irradiator has to be hand held and the technician is quite close to it, (ii) the average number of hours usually necessary to realize the control of the monitors fixed in the facilities of 4.7 hours/year (iii) the dose limit of $20 \mathrm{mSv} /$ year and (iv) the eventuality that the technician could be exposed to other sources of radiation, the decision to establish the maximum dose should be conservative.

For radiation protection proposes it is more useful to consider the dose at the distance of $10 \mathrm{~cm}$. At this distance, the naked source originates an ambient dose equivalent rate of $1.68 \mathrm{mSv} / \mathrm{h}$. Taking in to account the annual dose limits and considering the average number of hours necessary to realize the metrological control, the value of $4.26 \mathrm{mSv} / \mathrm{h}$ for the dose rate limit is obtained. Applying an arbitrary factor in order to decrease the dose value 50 times obtains the value for the dose rate at 10 $\mathrm{cm}$ of $0.085 \mathrm{mSv} / \mathrm{h}$.

So, the design of the irradiator was made in order to reduce the ambient dose equivalent from $1.68 \mathrm{mSv} / \mathrm{h}$ to $0.085 \mathrm{mSv} / \mathrm{h}$ at $10 \mathrm{~cm}$ of the surface of the irradiator. The methodology for the calculus was based on the iterative method presented by Turner [5].

Considering the case of narrow-beam geometry, the initial estimate of the shielding required to reduce the ambient dose equivalent of the above values leads to a number of relaxation lengths, $\mu x$, needed to satisfy the relation,

$$
e^{-\mu x}=\frac{0.085}{1.68}=0.050
$$

or $\mu \mathrm{x}=2.996$. According to the table for dose buildup factor B for a point isotropic source [3], the buildup factor for the energy of the gamma emission of ${ }^{137} \mathrm{Cs}$, $0.662 \mathrm{MeV}$ and for this number of relaxation lengths, is about 1.7.In order to maintain the reduction of 0.05 the same when the buildup factor is used, the number of relaxation lengths in the exponential must be increased. So, the number $z$ of added relaxation lengths that compensates a build factor of 1.7 is given by $e^{-z}=$ $1 / 1.7$, which means $z=0.531$.

So, the estimated shield thickness increases to 3.527 relaxation lengths corresponding to a new value for $\mathrm{B}$, of 1.85 and consequently a new $z=\ln 1.85=0.615$. This value originates a shield thickness of $2.996+0.615=3.611$ relaxation lengths. For $\mu x=3.611$, the new $B$ is 1.90 . So, the reduction factor with the buildup included is

$$
B e^{-\mu x}=1.90 e^{-3.611}=0.051
$$

which is practically identical with eq. (1). In any case the higher number of relaxation lengths will be used, $\mu \mathrm{x}=3.611$.

The mass attenuation coefficient for gamma energy of ${ }^{137} \mathrm{Cs}$ is $\mu / \rho=0.105 \mathrm{~cm}^{2} \mathrm{~g}^{-1}$. With $\rho=11.4 \mathrm{~g} \mathrm{~cm}^{-3}$ for lead obtains the value of $\mu=1.197 \mathrm{~cm}^{-1}$. Finally, the required thickness of lead shielding is $x=3.611 / 1.197=3.0 \mathrm{~cm}$
The design of the irradiator with a cylindrical shape, made of lead should have the minimum thickness of the walls of $3 \mathrm{~cm}$. Its several parts are shown in the figure 2 and the schematic design is shown in figure 3.

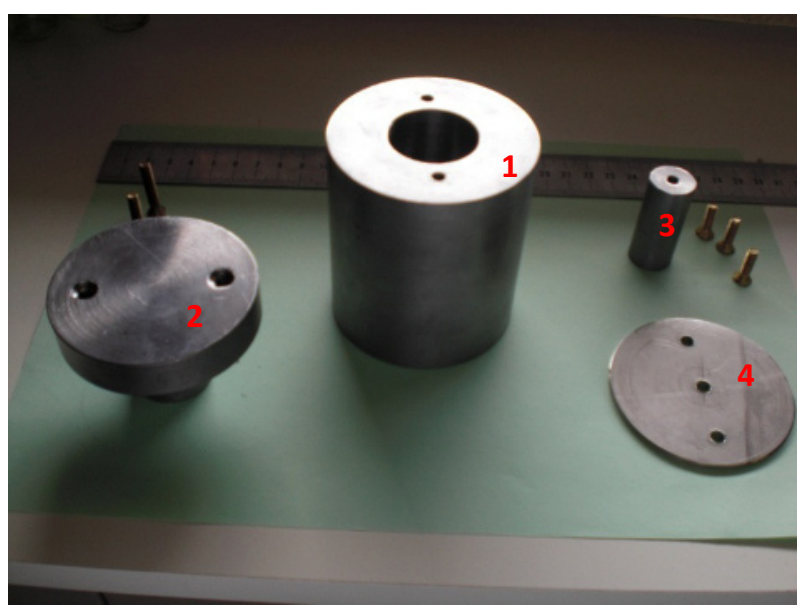

Figure 2.The several components of the irradiator.

1- Main body, 2-Removable cover, 3- Cylinder of lead, 4-Lid.

After the construction of the irradiator, the first operation was to introduce the removable cover, part 2 in figure 2 , in its position. Only when the irradiator is being used for metrological control it should be removed.

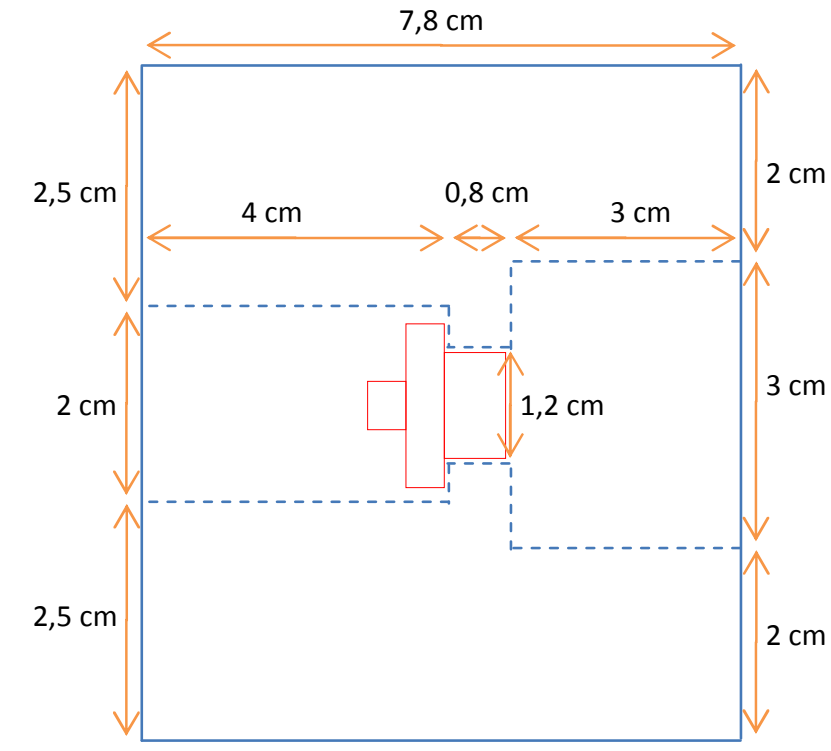

Figure 3. The schematic design of the main body of the irradiator with the source.

After that the source was introduced by the rear side of the irradiator. The cylinder of lead, part 3 in figure 2, was then also introduced. The lid, part 4, is then fixed. The radiation beam coming out of the irradiator without the removable cover is schematically represented in figure 5 . 




Figure 4. Lateral view of irradiator with a mini-laser tied to it



Figure 5.Schematic representation of the gamma beam coming out directly from the source.

\section{Characterization of the irradiator}

The characterization of the irradiator was realized considering two points of view. First considering the radiation protection characterization and secondly the radiation beam properties.

\subsection{Radiation protection characterization}

The operational quantity ambient dose equivalent, $H^{*}(10)$, was used to evaluate the performance of the irradiator in terms of radiation protection. To access to the ambient dose equivalent, the equipment used was a Babyline 31.

The dose rate values are expressed in $\mathrm{rad} / \mathrm{h}$ units. The dose was measured along various directions (see fig.6) at several distances from the irradiator. During these measurements the removable cover was in place. The dose values were converted in SI units, for Gy/h with the conversion factor of $1 \mathrm{rad}=10 \mathrm{mGy}$.

For each position 5 measurements were done. The measurements were done from $1 \mathrm{~m}$ to $10 \mathrm{~cm}$ distance to the irradiator. The background was also measured. Its value was $0.32 \mu \mathrm{Gy} / \mathrm{h}$. The results obtained are shown in figure 7 .

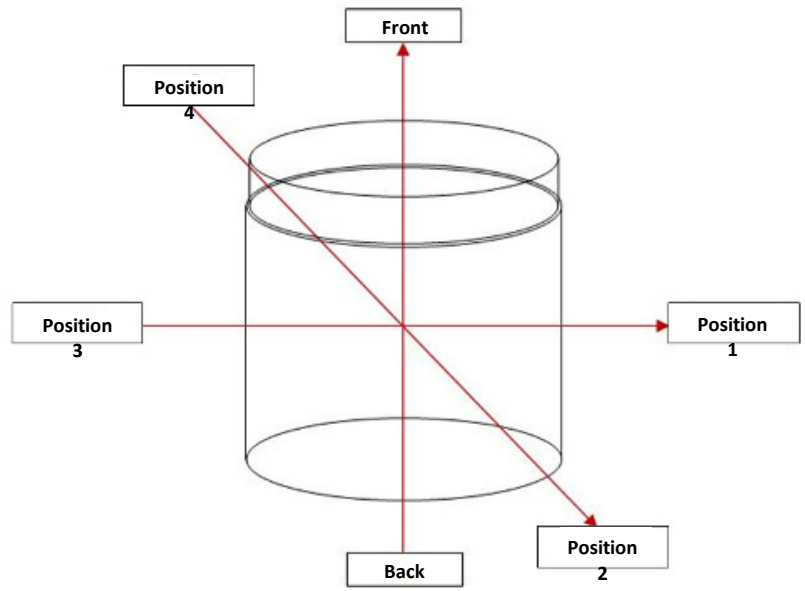

Figure 6.The axis where measurements were done.

It is possible to identify two main sets of values. One set encompasses the measurements taken on the 5 axis, axis 1, 2, 3, 4 and 5 with values slightly different among them; the second set with the measurements taken on the front axis with values rather different, lower, than the first ones. Such is due to the higher thickness of lead crossed by the radiation in this direction.

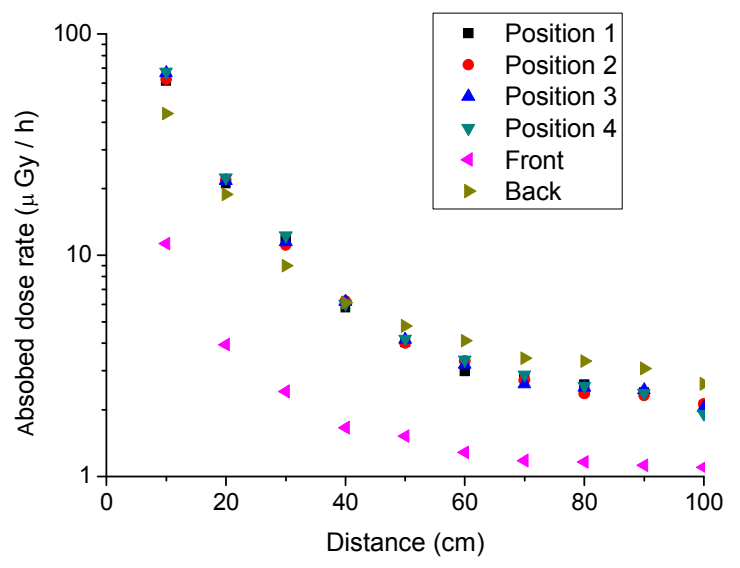

Figure 7.Absorbed dose as a function of the distance to the irradiator for several axis.

Due to the cylindrical symmetry, the values on 4 first axes are very similar and its average value for $10 \mathrm{~cm}$ distance is $64.63 \mu \mathrm{Gy} / \mathrm{h} \pm 3.10 \mu \mathrm{Gy} / \mathrm{h}$. The corresponding ambient dose equivalent is $0.078 \mathrm{mSv} / \mathrm{h} \pm 0.004 \mathrm{mSv} / \mathrm{h}$. This value shows a good agreement with the value estimated $(0.085 \mathrm{mSv} / \mathrm{h})$ from the calculus for the design of the irradiator.

\subsection{Radiation beam characterization}

The study of the profile of the radiation beam was performed by two different ways, the experimental method and the Monte Carlo simulation method. For experimental measurements was used the PTW 23361 ionizing chamber. It is a vented sensitive volume of 30 $\mathrm{cm}^{3}$, a diameter of $31 \mathrm{~mm}$ and a length of $51 \mathrm{~mm}$. An acrylic build-up cap with $3 \mathrm{~mm}$ wall thickness was used. Associated to this chamber the PTW UNIDOS electrometer was used. 
The Monte Carlo simulations studies were realized using the general Monte Carlo code, the MCNPX, version 2.7.0 [6]. Experimental and Monte Carlo simulation results were obtained for 3 distances from the irradiator, $20 \mathrm{~cm}$, $30 \mathrm{~cm}$ and $40 \mathrm{~cm}$.

The experimental measurements were carried out on the quantity electric charge. The acquisition time was of $60 \mathrm{~s}$, $120 \mathrm{~s}$ and $180 \mathrm{~s}$ and depends on the value of measurand. The atmospheric pressure and temperature were also registered in order to correct the measurements of the ionizing chamber for reference environmental conditions. For each position, 5 measurements were taken. Each profile determination was done starting with the ionizing chamber centred with the radiation beam and then moved the chamber $2 \mathrm{~cm}$ each time on the horizontal direction, (to the right and to the left) and on the vertical direction (up and down).

The MC determinations were done with the input of the MCNPX code with the data corresponding to the irradiator, its drawing, material and density, and to the ionizing chamber, introduced as a simplified model, and to the energy of the source, $0.662 \mathrm{MeV}$. The positions of the chamber-irradiator in the simulation study reproduced the positions on the experimental determinations. The quantity calculated was the air kerma in pGy/particle using the tally F6, specific of the MCNP family codes. As the simulated irradiator has a perfect cylindrical symmetry only a profile (horizontal or vertical) is obtained. The results are shown in figures 8,9 and 10 .

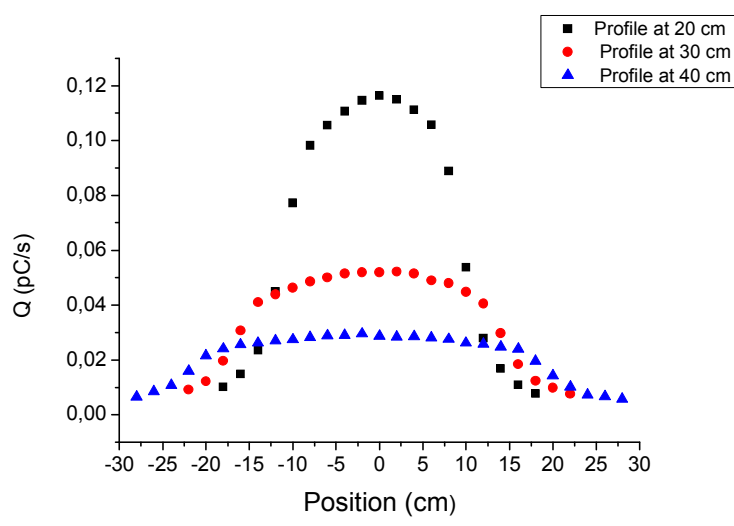

Figure 8. Experimental horizontal profiles of the beam for distances of $20 \mathrm{~cm}, 30 \mathrm{~cm}$ and $40 \mathrm{~cm}$.



Figure 9.Experimental vertical profiles of the beam for distances of $20 \mathrm{~cm}, 30 \mathrm{~cm}$ and $40 \mathrm{~cm}$.

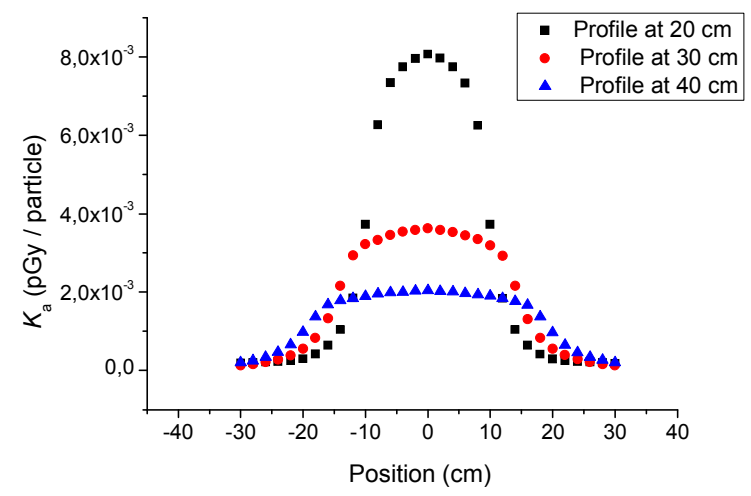

Figure 10. Monte Carlo simulations profiles of the beam for distances of $20 \mathrm{~cm}, 30 \mathrm{~cm}, 40 \mathrm{~cm}$ and $100 \mathrm{~cm}$.

As expected, the full width at half maximum (FWHM) is a linear function on the distance for both profiles. Although there is a good agreement between the two set of values, experimental and simulations results, it is possible to identify a slightly shift of the experimental data as compared with the simulation data. As example, is shown in figure 11 both profiles for $30 \mathrm{~cm}$. For this distance, the FWHM for experimental and simulations data is $28.8 \mathrm{~cm}$ and $31.4 \mathrm{~cm}$, respectively. This is probably due to a deviation from cylindrical symmetry on the position of the source inside the irradiator. However from a practical point of view this deviation is not very important. 


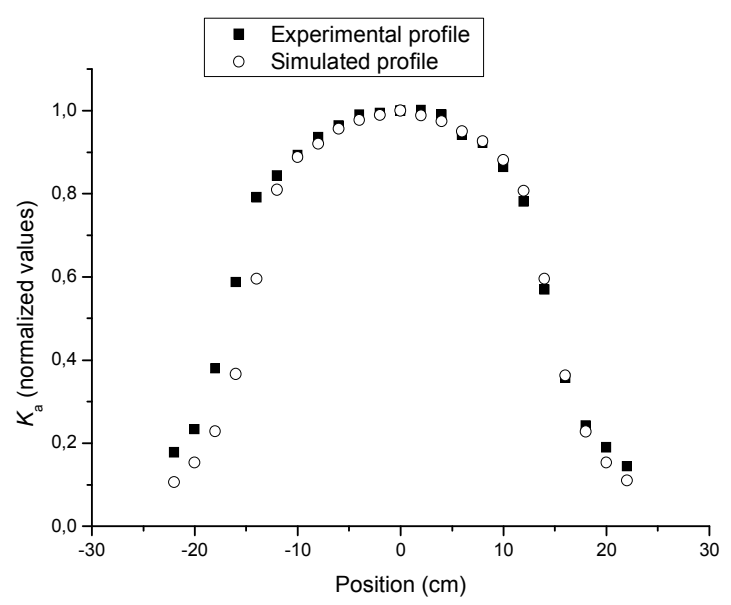

Figure 11. Experimental and simulated profiles for $30 \mathrm{~cm}$ distance.

The results obtained with the Monte Carlo code and its agreement with the experimental values allows concluding that the methodology used for the simulation is valid.

\section{Dosimetry}

The dosimetry is a key outcome of the project and construction of the irradiator. The ambient dose equivalent rate values determined during the dosimetry will be the reference values used to metrological control the installed monitors of the facilities. In addition to the experimental dosimetry the computational dosimetry by Monte Carlo simulations was also performed.

\subsection{Methodology}

The dosimetry followed the ISO 4037-2 [7], and was done using two different volume chambers, a smaller chamber with $30 \mathrm{~cm}^{3}$ sensitive volume and a 1 litre ionization chamber. Two ionizing chambers and a PTW UNIDOS electrometer were used: a smaller chamber, the PTW 23361 with $30 \mathrm{~cm}^{3}$ volume and a larger chamber, the PTW 32002 with a sensitive volume of $1000 \mathrm{~cm}^{3}$.

The measurements were done in the charge mode. The chambers were positioned at the central axis of the beam. The range of the measurements was from $10 \mathrm{~cm}$ to 110 $\mathrm{cm}$ for the smaller chamber and $20 \mathrm{~cm}$ to $160 \mathrm{~cm}$ for the larger chamber with steps of $10 \mathrm{~cm}$. The acquisition time was $60 \mathrm{~s}, 120 \mathrm{~s}$ and $180 \mathrm{~s}$ and depends on the value of measurand.

The background and leakage measurements were done and subtracted to the measurements with ionizing radiation. After these initial measurements, the chamber is properly positioned, it was removed the cover and the corresponding value of the charge $M(\mathrm{C} / \mathrm{s})$ was registered.

To determine the $H^{*}(10)$ value the following equation was applied:

$$
H^{*}(10)=M \times N_{P, T} \times N_{K a} \times C C \quad[S v / s](3)
$$

where $N_{P, T}$ is the correction factor for reference pressure and temperature, $N_{K a}$ is air kerma calibration coefficient of the chamber expressed in units of $[G y / C]$ and $C C$ is the conversion coefficient of $K a$ to $H^{*}(10)$ for energy of $0.662 \mathrm{MeV}$ express in units of $[S v / G y]$.

To each measurement $M$, including the background measurement, is applied the equation above. The background value is subtracted to all $H^{*}(10)$ values.

For the Monte Carlo simulations with the MCNPX code, the input includes the ionizing chamber and the irradiator without the cover. The distances between chamber and irradiator are the same as used in experimental setup. The calculated quantity is the air kerma, express in[Gy/particle], using the tally F6. Applying the $C C$ on obtain the quantity $H^{*}(10)$ expressed in Sv/particle.

In order to be able to compare the results with the experimental ones, the normalization factor was calculated using all the values of both methods and determined the ratio between the two set of values. The average value was adopted as the normalization factor.

\subsection{Results}

The results of the dosimetry are shown on figures 12 and 13.

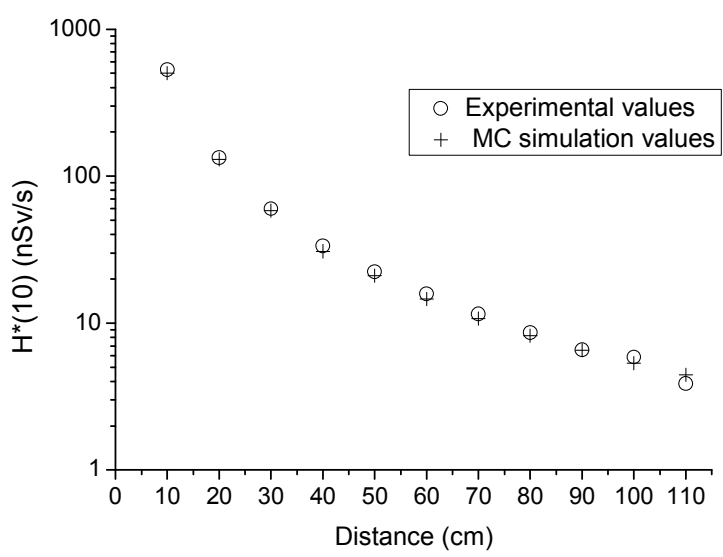

Figure 12. Experimental and Monte Carlo simulations results relatives to the ionizing chamber PTW 23361.

The figure 12 shows the results of the experimental and Monte Carlo simulations relatives to the smaller ionizing chamber, the PTW 23361 chamber.

The agreement between the two set of values is good. Both sets of values are adjusted by a function $y=a x^{b}$

The $b$ values are -1.970 and -1.986 for function adjusted to the Monte Carlo values and experimental values, respectively, which allows to concluding that the law of the inverse of square root is followed very closely. The uncertainty budget has been determined for each distance according to the GUM [8]. On the table 1 are shown all the values of $H^{*}(10)$ for this chamber, and their uncertainties. 
Table 1. Values of $H^{*}(10)$ for the PTW 23361 chamber, and their uncertainties.

\begin{tabular}{|c|c|c|c|}
\hline $\begin{array}{c}\text { Distance } \\
(\mathbf{c m})\end{array}$ & $\begin{array}{c}\mathbf{H}^{*}(\mathbf{1 0}) \\
(\mathbf{n S v} / \mathbf{s})\end{array}$ & $\begin{array}{c}\text { Uncertainty } \\
(\mathbf{n S v} / \mathbf{s})\end{array}$ & $\begin{array}{c}\text { RelativeUncertainty } \\
(\mathbf{\%})(\boldsymbol{k}=\mathbf{1})\end{array}$ \\
\hline 20 & 118.40 & 2.44 & 2.06 \\
\hline 30 & 55.16 & 1.14 & 2.06 \\
\hline 40 & 31.47 & 0.66 & 2.09 \\
\hline 50 & 20.25 & 0.42 & 2.08 \\
\hline 60 & 14.29 & 0.32 & 2.22 \\
\hline 70 & 10.54 & 0.28 & 2.66 \\
\hline 80 & 9.26 & 0.24 & 2.60 \\
\hline 90 & 6.46 & 0.20 & 3.09 \\
\hline 100 & 5.33 & 0.18 & 3.38 \\
\hline 110 & 4.00 & 0.16 & 4.00 \\
\hline 120 & 3.11 & 0.20 & 6.41 \\
\hline 130 & 2.89 & 0.13 & 4.57 \\
\hline 140 & 2.65 & 0.13 & 4.82 \\
\hline 150 & 2.56 & 0.29 & 11.44 \\
\hline 160 & 2.07 & 0.30 & 14.67 \\
\hline & & & \\
\hline
\end{tabular}

The figure 13 shows the results of the experimental and Monte Carlo simulations relative to the larger ionizing chamber, the PTW 32002 chamber. The criterion for normalization is the same as applied previously.

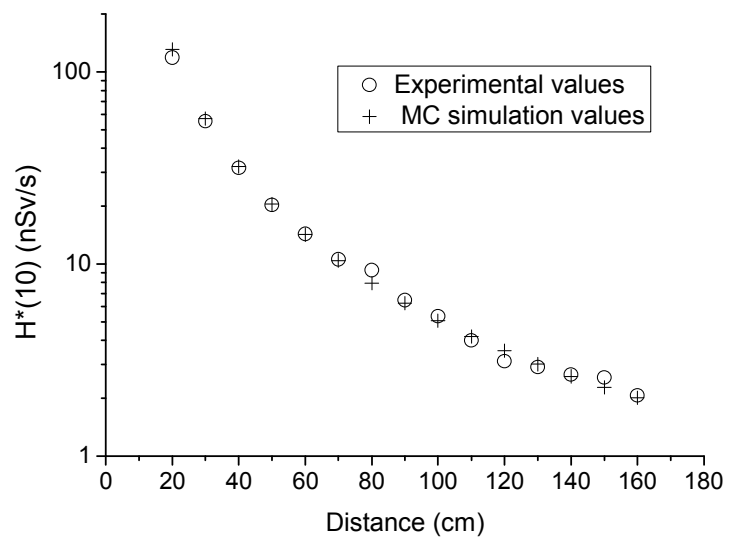

Figure 13. Experimental and Monte Carlo simulations results relatives to the ionizing chamber PTW 32002.

The agreement between the two set of values is good. Both sets of values are adjusted by the functiony $=a x^{b}$.

The $b$ values are -2.023 and -1.916 for Monte Carlo and experimental values, respectively. The law of the inverse of square root is also followed very closely. In table 2 all the values of $H^{*}(10)$ for this chamber, and their uncertainties are shown.

Looking at tables 1 and 2 it is possible to observe that within a distance from the irradiator to the chamber up to $80 \mathrm{~cm}$ the $H^{*}(10)$ values are higher for PTW23361 than for the PTW 32002. In fact, for the smaller chamber the ratio direct photons/scattered photons reaching the chamber is higher than for the larger chamber leading to the higher $H^{*}(10)$ values. For lengthier distances than 80 $\mathrm{cm}$ the uncertainties increase and there is no clear trend.

\subsection{Study of the influence of the wall supporting the monitor}

Sometimes the monitors to be controlled in situ are fixed to a wall as has been already stated. This wall can influence the measurements because it is very near to the detector of the monitor originating scattering of the photons which can reach the detector. In order to quantify this influence a Monte Carlo study was realized using only the smaller chamber. The wall has surface of $2 \mathrm{~m} \times$ $2 \mathrm{~m}$ and a thickness of $20 \mathrm{~cm}$. Two densities were considered. The density of $2.3 \mathrm{~g} / \mathrm{cm}^{3}$ and the density of $1.5 \mathrm{~g} / \mathrm{cm}^{3}$. The chemical composition is the same.

So, the simulated setup is similar to that used for dosimetry with the ionizing chamber PTW 23361 but now a wall is simulated behind the chamber. The chamber is centred with the wall. Relative difference between the results with and without the wall are shown in figure 14

For all the distances considered the geometry with the wall always shows higher values. For the higher density wall, at $10 \mathrm{~cm}$ distance the difference between the case with wall or without wall, is $2.9 \%$ and at $200 \mathrm{~cm}$ distance the difference is $14.6 \%$. For the lower density wall the results are very similar showing differences of $2.4 \%$ and $12.2 \%$ for the same distances. This increase in the value of $H^{*}(10)$ with the distance is due to the increase of the area of the wall directly reached by the photons emitted by the source thereby leading to a greater contribution of scattered radiation reaching the detector.

Table 2. Values of $H^{*}(10)$ and their uncertainties for the PTW 32002 chamber.

\begin{tabular}{|c|c|c|c|}
\hline $\begin{array}{c}\text { Distance } \\
(\mathbf{c m})\end{array}$ & $\begin{array}{c}\mathbf{H}^{*}(\mathbf{1 0}) \\
(\mathbf{n S v} / \mathbf{s})\end{array}$ & $\begin{array}{c}\text { Uncertainty } \\
(\mathbf{n S v} / \mathbf{s})\end{array}$ & $\begin{array}{c}\text { RelativeUncertainty } \\
(\mathbf{\%})(\boldsymbol{k}=\mathbf{1})\end{array}$ \\
\hline 10 & 530.24 & 11.52 & 2.17 \\
\hline 20 & 133.56 & 2.91 & 2.18 \\
\hline 30 & 59.96 & 1.32 & 2.21 \\
\hline 40 & 33.57 & 0.78 & 2.32 \\
\hline 50 & 22.36 & 0.57 & 2.57 \\
\hline 60 & 15.79 & 0.43 & 2.70 \\
\hline 70 & 11.53 & 0.33 & 2.88 \\
\hline 80 & 8.61 & 0.24 & 2.83 \\
\hline 90 & 6.57 & 0.24 & 3.63 \\
\hline 100 & 5.88 & 0.18 & 3.06 \\
\hline 110 & 3.88 & 0.27 & 7.01 \\
\hline
\end{tabular}




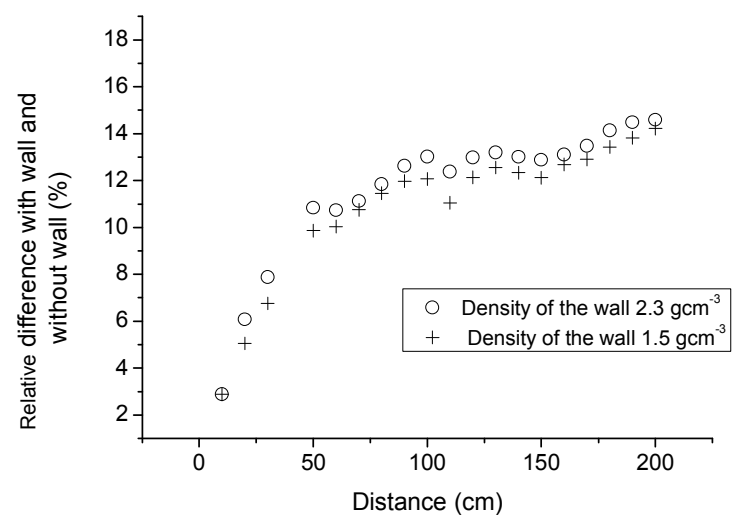

Figure 14. Influence of the wall in the dosimetric Monte Carlo results.

The correction factors applied to the $H^{*}(10)$ are shown in table 3 .

Table 3. Wall correction factors for $H^{*}(10)$.

\begin{tabular}{|c|c|c|}
\hline $\begin{array}{c}\text { Distance } \\
\text { (cm) }\end{array}$ & $\begin{array}{c}\text { High density } \\
\text { Wall correction } \\
\text { factor }\end{array}$ & $\begin{array}{l}\text { Low density wall } \\
\text { correction factor }\end{array}$ \\
\hline 10 & 1.029 & 1.024 \\
\hline 20 & 1.061 & 1.042 \\
\hline 30 & 1.079 & 1.054 \\
\hline 50 & 1.108 & 1.082 \\
\hline 60 & 1.107 & 1.085 \\
\hline 70 & 1.111 & 1.089 \\
\hline 80 & 1.118 & 1.096 \\
\hline 90 & 1.126 & 1.096 \\
\hline 100 & 1.13 & 1.095 \\
\hline 110 & 1.124 & 1.093 \\
\hline 120 & 1.13 & 1.103 \\
\hline 130 & 1.132 & 1.107 \\
\hline 140 & 1.13 & 1.104 \\
\hline 150 & 1.129 & 1.102 \\
\hline 160 & 1.131 & 1.107 \\
\hline 170 & 1.135 & 1.11 \\
\hline 180 & 1.141 & 1.115 \\
\hline 190 & 1.145 & 1.118 \\
\hline 200 & 1.146 & 1.122 \\
\hline
\end{tabular}

\section{Conclusions}

The design and construction of an irradiator to allow to carryout in situ the metrological control of installed radiation protection monitors measuring the ambient dose equivalent was done. The dose at $10 \mathrm{~cm}$ distance from the irradiator was established in a very conservative way and it is very close to the expected value. The profile determinations allowed to knowing the variation of the FWHM with the distance and validate the methodology used for the Monte Carlo simulation studies.

Dosimetry was realized using two ionizing chambers.

The results are different at a distance of $80 \mathrm{~cm}$ which can be justified by the different contribution of the scattered photons reaching the detector.

Finally, the influence for the presence of a wall behind the monitor was studied and corrections factors were determined. They can increase the $H^{*}(10)$ values until 15 $\%$.

The irradiator is ready to perform the in situ metrological control of installed ambient dose equivalent monitors.

This work was supported by the FCT funded project PTDC/AAC-AMB/121375/2010 - VADOSE

\section{References}

1. Council Directive 96/29/Euratom of 13 May 1996. Laying down basic safety standards for the protection of the health of workers and the general public against the dangers arising from ionizing radiation. Official Journal, No. L159, 1-114.

2. International Standard, IEC 60532. Radiation protection instrumentation - Installed dose rate meters, warning assemblies and monitors - $\mathrm{X}$ and gamma radiation of energy between $50 \mathrm{keV}$ and $7 \mathrm{MeV}$. Edition 3.0. 2010

3. ISO 4037-1:1996, $\mathrm{X}$ and gamma reference radiation for calibrating dosemeters and dose rate meters and for determining their response as a function of photon energy - Part 1: Radiation characteristics and production methods.

4. ISO 4037-3:1997, $\mathrm{X}$ and gamma reference radiation for calibrating dosemeters and dose rate meters and for determining their response as a function of photon energy - Part 3: Calibration of area and personal dosemeters and the measurement of their response as a function of energy and angle of incidence.

5. Turner, James E., Atoms, Radiation, and Radiation Protection. Second Edition. John Wiley \& Sons, Inc. 1995

6. Pelowitz, D., MCNPX User's Manual. Los Alamos National Laboratory Report, LA-CP-05-0368. 2005

7. ISO 4037-2:1997, $\mathrm{X}$ and gamma reference radiation for calibrating dosemeters and dose rate meters and for determining their response as a function of photon energy - Part 2: Dosimetry for radiation protection over the energy ranges $8 \mathrm{keV}$ to $1,3 \mathrm{MeV}$ and $4 \mathrm{MeV}$ to $9 \mathrm{MeV}$.

8. Evaluation of measurement data - Guide to the expression of uncertainty in measurement. JCGM 2008. 